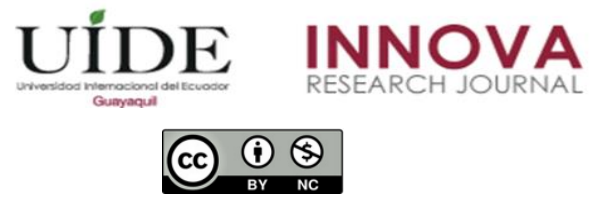

INNOVA Research Journal, ISSN 2477-9024

(Septiembre-Diciembre 2019). Vol. 4, No.3.1 pp. 126-134

DOI: https://doi.org/10.33890/innova.v4.n3.1.2019.1082

URL: http://revistas.uide.edu.ec/index.php/innova/index

Correo: innova@uide.edu.ec

\title{
Fortalecimiento del desarrollo turístico sostenible en los humedales de Ventanilla, departamento de Lima, Perú
}

\section{Strengthening sustainable tourism development in the Ventanilla wetlands, Department of Lima, Peru}

Mondragón Mondragón Duber Ysai

ORCID: https://orcid.org/0000-0002-3778-2718

Salvador Garcia Claribel Rosario

Universidad Norbert Wiener, Perú

Autor para correspondencia: dubermondragon1993@gmail.com; claricielo@ hotmail.com Fecha de recepción: 12 de julio de 2019 - Fecha de aceptación: 16 de octubre de 2019

\section{Resumen}

El estudio se realizó en el distrito de Ventanilla, con el objetivo de elaborar un plan de fortalecimiento del desarrollo sostenible en los humedales de Ventanilla, Lima, aportando al cuidado y preservación del área natural. La investigación es de enfoque mixto, sintagma holístico y de tipo proyectiva. Para hacer el diagnostico se aplicó encuestas tomando como muestra a 116 pobladores locales y 25 visitantes del recurso, también se entrevistó a 3 profesionales vinculados al turismo en la zona. Los resultados indicaron que en la localidad no existe responsabilidad ambiental, en cuanto al recurso las acciones que se están gestionando son insuficientes para el cuidado de toda el área natural y evitar la contaminación. Por otra parte, el recurso carece de infraestructura turística adecuada para los recorridos, lo cual hace que el turista no reciba una grata visita. Finalmente, se planteó desarrollar un plan basado en las prácticas de ecoturismo, junto a talleres dirigidos a pobladores para lograr un compromiso ambiental, realizar campañas de limpieza, elaborar un circuito basado en la observación de la biodiversidad para evitar daños a la naturaleza, acondicionar el lugar para una visita satisfactoria, además incentivar a los comuneros a participar en actividades de artesanía.

Palabras clave: contaminación; invasión; preservación; infraestructura turística; responsabilidad ambiental

\begin{abstract}
:
The study was released in the district of Ventanilla, with the objective of developing a plan to strengthen sustainable development in the wetlands of Ventanilla, Lima, contributing to the care and preservation of the natural area. The research is mixed focus, holistic syntagm and projective type. To make the diagnosis, surveys were applied, it was taken as sample to 116 local inhabitants and 25 visitors of the resource, also interviewed 3 professionals linked to tourism in the area. The results indicated that in the locality there is not environmental responsibility, as for the resource, the actions that are being managed are insufficient for the care of of all the natural área and avoid pollution. On the other hand, the resort lacks adequate tourist infrastructure for the tours, which means that tourists do not receive a pleasant visit. Finally, it was proposed to develop a plan based
\end{abstract}


on ecotourism practices, alongside workshops aimed at residents to achieve an environmental commitment, realize cleaning campaigns, elaborate a circuit based on observing the biodiversity to avoid damage to nature, condition the place for a satisfactory visit with signage, railings and defined paths, besides encourage to community members to participate in activities of crafts.

Key words: pollution; invasion; preservation; tourist infrastructure; environmental responsibility

\section{Introducción}

Debido a los constantes cambios de la industria turística, las necesidades del visitante también han ido evolucionando, por lo que se generan nuevas tendencias entorno al turismo que estén acorde con las expectativas de los consumidores, pero siempre respetando ciertos estándares de calidad que permita realizar turismo con responsabilidad contribuyendo con la sostenibilidad del lugar donde se desarrolla, sin causar daños al medio ambiente y que los beneficios percibidos por la actividad turística sean equitativos con los integrantes de las comunidades. Por ello se busca diferentes alternativas que permitan el desarrollo sostenible del lugar donde se llevara a cabo el turismo, a nivel internacional tenemos como ejemplo a Colombia y Costa Rica son países que han logrado el desarrollo de varias de sus localidades mediante actividades de ecoturismo, tienen una imagen de destino turístico consolidada en base a sus atractivos naturales, lo cual aporta al cuidado del medio ambiente, creando grandes oportunidades de trabajo para prestadores de servicios y ayudando también al crecimiento económico de sus pobladores locales (Efe verde, 2018; Crhoy, 2018).

A nivel nacional, Perú es un país dueño de una gran variedad en flora y fauna, por lo que es considerado como una de las mejores opciones en todo el mundo para realizar actividades que están relacionadas con la naturaleza, como la observación de aves, protegiendo así el medio ambiente de la mano con el desarrollo local (PROMPERU, 2018). Por tal motivo nuestro país dispone de todo el potencial para desarrollar este tipo de actividades buscando la inclusión de la población para mejorar su estilo de vida, uno de los lugares que cuenta con todas las características es los humedales de Ventanilla, área natural poseedor de diversidad de avifauna, flora y formas geológicas que se pueden aprovechar para la observación del visitante.

El humedal consta de 275. 45 hectáreas, situado en una zona desértica en la parte centro al occidente del distrito de Ventanilla - Callao, es un área de conservación bajo la protección del gobierno regional del Callao, tiene un ecosistema que abarca lagunas, habitad en avifauna con más de 121 familias diferentes de aves residentes y migratorias y otros tipos de vida silvestre, es posible encontrar más de 20 tipos de comunidades vegetales, siendo las más predominantes conocidas como la planta del junco, la totora y el carrizo, las mismas que son utilizadas en la elaboración artesanal, el área es poseedor de un paisaje único, rodeado transformaciones geológicas, cuevas, entre otras que son producto de la erosión geonatural y alrededor del lugar se encuentra la playa costa azul. (Gobierno Regional del Callao, 2009).

A pesar del gran potencial de este recurso, no tiene trascendencia turística, se encuentra olvidado y en parte depredado por la expansión urbana, lo que demuestra que no existe una gestión por parte de las autoridades encargadas que busquen estrategias para el desarrollo y la difusión del recurso, debido a ello no hay interés por los operadores de servicios turísticos. 
Según estudios realizados existen acontecimientos que dañan la imagen del lugar, lo cual se ve reflejado en la contaminación que existe a los alrededores por la falta de cuidado y al desconocimiento de la importancia turística por parte de los pobladores, del mismo modo el nivel de pobreza que existe alrededor es eminente, la población no dispone de los servicios básicos (Medialab, 2018). Por tal razón es necesario potencializar estas actividades de naturaleza involucrando a la comunidad para el surgimiento económico del poblador local.

Para ello nos basamos en la teoría de las tres dimensiones del desarrollo sostenible, la cual establece diferentes puntos importantes que se deben seguir para un crecimiento sustentable, es indispensable tener en cuenta el tema ambiental y fomentar la inclusión de la sociedad enfocándose al uso correcto de los recursos satisfaciendo una necesidad en las comunidades locales o visitantes sin causar daños al planeta ya que es legado para las generaciones futuras (Artaraz, 2002; Bermejo, 2014).

Para alcanzar un crecimiento turístico sustentable, también es necesario la participación de la población, dado que según la teoría del desarrollo local territorial para lograr el progreso de una localidad se requiere del trabajo en conjunto con todos los integrantes del sistema turístico, logrando que los beneficios económicos percibidos por la actividad turística sean equitativos con la comunidad (Quintero 2008; Mantero, 2004; Vásquez 2000).

Debemos comprender que toda estrategia que se busque para el desarrollo de un lugar determinado debe ser gestionado respetando su cultura y costumbres de la localidad, usar al turismo como un medio para trasmitir valores que puedan ser tomados como herramientas para generaciones futuras. La teoría del sistema turístico da un enfoque a la actividad turística como un sistema que sirve de intercomunicador entre diferentes naciones fomentando la unión de las mismas, intercambiando valiosa información de impacto positivo para la sociedad, sin generar acontecimientos que dañen las comunidades (Cuervo, 1967). Asimismo, Leiper plantea un modelo como herramienta para la gestión en base a los componentes del sistema turístico, señala como agentes principales el área donde se llevará a cabo la actividad, los visitantes y las empresas que operan servicios turísticos, la interrelación que existe entre estos elementos es influenciada por el ambiente externo (Panosso y Lohamann, 2012).

Ugaz (2018) analizó las oportunidades de crecimiento económico sustentable en la ciudad de Amazonas, Perú; en la cual se demuestra que realizar actividades relacionadas con el medio ambiente donde se trabaje en conjunto con las autoridades encargadas y los mismos habitantes aledaños es una alternativa para sacar adelante las zonas rurales. A nivel regional Críales (2014), sostiene en su investigación la necesidad de implementar instalaciones turísticas, pero causando el menor impacto negativo en las áreas naturales, propuso un centro de interpretación como parte del proceso de mejora de la infraestructura en la albufera de Huacho en el departamento de Lima, lo cual ayudaría con el desarrollo del ecoturismo generando beneficios para los integrantes de la localidad.

Por esta razón se debe plantear un plan en los humedales de Ventanilla que incluya el acondicionamiento turístico debido a que el recurso solo dispone de instalaciones básicas, por lo tanto, si se quiere lograr el progreso turístico y de la zona es necesario incrementar las visitas 
mediante el servicio de calidad y para ello se debe contar con instalaciones adecuadas que permitan realizar los recorridos satisfactoriamente y lograr una grata visita.

Es importante comprender que el desarrollo turístico sostenible se refiere a toda actividad que tiene presente las consecuencias actuales y los efectos futuros que ello puede ocasionar en el aspecto económico, medioambiental o en la sociedad, lo cual permitirá cubrir satisfactoriamente una necesidad (Organización Mundial de Turismo, 1993).

\section{Las subcategorías consideradas fueron las siguientes}

Preservación del medio ambiente, se relaciona con la buena gestión ambiental por ello se define como la acción de defensa, que tiene en cuenta la protección de los recursos del medio ambiente, recomendando la inclusión de la ciudadanía en las actividades, enfocándose hacia el uso sostenible con el tiempo (Secretaria de Turismo de la Nación, 2007).

Instalaciones turísticas, son los elementos físicos vinculados directamente con el atractivo turístico, los cuales van a permitir la fácil accesibilidad del turista al realizar el recorrido y lograr la satisfacción del mismo (Ministerio de Comercio Exterior y Turismo, 2011).

Calidad de vida, definido como la dimensionalidad de la condición en el entorno, que se consideran importantes para definir el crecimiento de las personas entorno a su estilo de vida, los cuales serán evaluados por las mismas personas si realmente le es beneficios para mejorar su desarrollo (Massam, 2002).

Por ende, para fines de esta investigación el objetivo principal fue diseñar un plan de ecoturismo que fortalezca el desarrollo turístico sostenible en los humedales de Ventanilla, departamento de Lima Perú; mientras que los objetivos específicos fueron diagnosticar la situación actual sobre actividad turística en la zona; determinar los factores que intervienen en el crecimiento económico sustentable de la comunidad; definir las estrategias influyentes para potencializar el desarrollo de los humedales de Ventanilla.

\section{Material y métodos}

El estudio se acoge al sintagma holístico con un enfoque mixto haciendo un análisis cuantitativo y cualitativo con el fin de obtener un mejor resultado. El tipo de investigación es proyectiva con el propósito de diseñar una propuesta en base a los resultados obtenidos para resolver situaciones determinadas, la cual podría aplicarse en un futuro (Hurtado, 2012). Es de nivel comprensivo basado en el método inductivo y deductivo. La categoría problema fue el desarrollo turístico sostenible y las sub categorías: Preservación del medio ambiente; instalaciones turísticas; calidad de vida de los pobladores. La población en estudio fueron habitantes de los asentamientos humanos de los humedales de Ventanilla, obteniendo una muestra de 116 pobladores locales y 25 visitantes del recurso.

Los datos que se han utilizado para esta investigación fueron por intermedio de encuestas, se aplicó un cuestionario de 18 preguntas bajo la escala de Likert, validado por expertos y confiabilidad. Además, se realizó una interpretación cualitativa usando como técnica la 
entrevista aplicada a 3 profesionales vinculados a la actividad turística en la zona. Para el análisis de resultados estadísticos se empleó el programa SPSS, posteriormente se triangulo la información mediante el Atlas. ti porque permite la codificación de datos para poder segmentar la información obtenida, relacionando definiciones y en base a ello construir una teoría o un diagnostico (Hernández, Fernández, y Baptista, 2014).

\section{Resultados}

En la interpretación de los datos cuantitativos con respecto a la preservación del medio ambiente, el $90 \%$ de los pobladores indica que en la comunidad no existe responsabilidad ambiental y un $10 \%$ manifiesta que si hay compromiso ambiental. Asimismo, en cuanto a los visitantes un $76 \%$ señalan que las acciones que han realizado en el atractivo de los humedales de Ventanilla y a los alrededores no son suficientes para controlar la contaminación. Con relación a la vigilancia y cuidado del recurso natural, un $56.9 \%$ de la población considera que es insuficiente para toda la cantidad de hectáreas que posee el lugar ya que gran parte del terreno está siendo depredado por los mismos habitantes aledaños.

Referente a las practicas ecológicas que se pueden desarrollar en los humedales, un $54.3 \%$ consideran la observación de flora y fauna como una buena alternativa para aportar al cuidado del medio ambiente. Por lo tanto, se puede determinar la falta de compromiso con el cuidado del medio ambiente en la localidad y que mediante las prácticas de turismo de observación de naturaleza en los humedales sería una forma de concientizar a la población y turistas apoyando al crecimiento económico del distrito.

En la sub categoría de instalaciones turísticas acerca de la señalización, el 88\% de las personas que visitaron el lugar consideran que la demarcación de los senderos y las señalizaciones no sean adecuadas para continuar con el recorrido, mientras que un $12 \%$ indica que es la correcta. Por ello al preguntarle sobre la implementación de instalaciones el $96 \%$ manifiesta que en necesario por seguridad y que faciliten la observación de flora y fauna del recurso. Por tal motivo resalta la necesidad de acondicionar adecuadamente la infraestructura para los recorridos, puesto que las instalaciones básicas que poseen actualmente los humedales no son suficiente para la satisfacción del visitante.

En relación a la sub categoría calidad de vida, el $90 \%$ de los pobladores señalan no percibir beneficios económicos que provengan de la actividad turística, pero existe un porcentaje del $10 \%$ que se beneficia mediante la actividad artesanal de la planta del junco que habita en los humedales. En cuanto al desarrollo local, se les formulo la pregunta si es estarían dispuestos a integrar un programa de desarrollo turístico sostenible, el 73.3\% manifiesta interés en participar en las actividades turísticas. En tal sentido existe la necesidad de implementar un plan de desarrollo donde involucre la comunidad para apoyar al sustento económico del poblador.

Asimismo, en la información obtenida por las entrevistar realizadas a los expertos, en cuanto se refiere a programas de conservación manifestaron que la municipalidad distrital únicamente organiza un evento conmemorativo en el día de los humedales para crear conciencia a la población, se llevan a cabo en el mismo humedal o también el auditorio municipal. Resaltaron el grave problema contaminación sobre todo en el sendero de cuevas generado por 
habitantes y bañistas de las playa, otra de las incidencias es la invasión del terreno con uno de los asentamientos humanos la Cooperativa Apurímac, ocasionan desmonte, arrojo basura, el ingreso informal al recurso debido a la falta de un cerco perimétrico que cubra toda el área, se menciona la importancia de contar con más personal de vigilancia y cuidado del recurso para evitar la depredación del terrero producto de la invasión de algunos comuneros, puesto que todas estas falencias generan daños a los espejos de agua.

Por otro lado, los entrevistados también mencionan que se requiere mejorar los servicios brindados de la mano con infraestructura que permita al turista desplazarse sin problema logrando obtener una grata visita, así como también expresan necesario la difusión del recurso para incrementar las visitas.

\section{Plan de fortalecimiento del desarrollo turístico sostenible}

Para que el turismo sea sostenible con el tiempo se basa en encontrar un equilibrio entre los beneficios económicos que se perciben de las acciones realizadas por la industria turística, la población y el respeto al ambiente con todos sus activos naturales sin afectar a la sociedad (Lalangui, Espinoza y Pérez, 2017).

Es necesario implementar un plan que potencialice los humedales de Ventanilla que sirva como herramienta de gestión en el crecimiento local y turístico, enfocado al cuidado del medio ambiente respetando las características del recurso que es el paisaje con todos los valores naturales que alberga para que los habitantes tomen conciencia de la importancia del recurso desarrollando una educación medioambiental.

El plan propuesto está basado en las prácticas ecológicas, por lo cual es importante entizar el concepto de ecoturismo que consiste en el desplazamiento del turista en áreas naturales actuando de manera responsable con el medio ambiente, sin afectar el bienestar de las comunidades. (The International Ecotourism Society, 2015). Los recursos naturales se deben manejar adecuadamente para no generar impactos negativos, aportando a las comunidades, puesto que al obtener crecimiento económico mejorará el estilo de vida local, para ello se estableció tres objetivos:

Objetivo 1: Sensibilizar a la población fomentado la conservación de los humedales de Ventanilla.

Actividades:

- Fomentar la conciencia ambiental mediante charlas informáticas a los habitantes de la comunidad y visitantes del recurso, enseñando el manejo adecuado de residuos.

- Convocar a universidades, colegios y pobladores locales para realizar campañas de limpieza ambiental en diferentes puntos críticos de contaminación en la zona.

- Desarrollar circuitos de observación de la biodiversidad del lugar, con una experiencia vivencial en los procesos de artesanía. Mostrar el valor natural del recurso mediante un centro fotográfico de los activos naturales de los humedales. 
Objetivo 2: Acondicionar turísticamente las instalaciones del recurso para un adecuado recorrido en el humedal.

Actividades:

- Potencializar la señalización, senderos del recorrido turístico protegiendo las áreas de reproducción.

- Mejorar el servicio brindado, formando guardaparques, guías voluntarios, contar con materiales para el turista que ayuden a la observación de la biodiversidad del humedal.

- Facilitar la información turística del recurso contando con un módulo informativo en la municipalidad, asimismo apoyarse de una plataforma virtual que vincule las campañas organizadas para cuidarlo y difundir su riqueza en biodiversidad.

Objetivo 3: Fomentar la participación de los pobladores en las actividades de desarrollo turístico sostenible.

Actividades:

- Incentivar el emprendimiento local, buscando oportunidades de crecimiento económico valiéndose de sus habilidades.

- Acciones para fortalecer la artesanía con la planta del junco, convocando a la población y promocionarlo mediante ferias.

- Elaborar programas de información para la etapa escolar, inculcando el valor de los humedales y lo beneficioso de cuidar el lugar.

\section{Conclusiones}

El recurso turístico los humedales de Ventanilla carece de protección, se encuentra en una lucha con la contaminación ambiental, además de la depredación del terreno por los invasores que habitan a los alrededores del terreno, falta infraestructura, ya que no se encuentran bien demarcados los senderos por donde el visitante hace el recorrido. Por esa razón se propone acondicionar turísticamente para seguridad del turista, además de las acciones de manejo de residuos tanto en el área natural como en los asentamientos humanos que lo rodean, informar desde la etapa escolar sobre lo inseguro de habitar en los terrenos de los humedales puesto que son movedizos para evitar la invasión se siga expandiendo.

Los factores que influyen en el desarrollo turístico sostenible de los humedales de Ventanilla es la conservación del medio ambiente, dado que para desarrollar actividades de naturaleza responsablemente es requisito tener en cuenta la preservación ambiental en el recurso y en la localidad. La calidad de vida local es otro de los elementos influyentes, debido al nivel de pobreza que existe a los alrededores de la zona, se busca la inclusión de los comuneros en el plan de desarrollo para mejorar su situación actual.

Finalmente se propone elaborar un plan basado en el ecoturismo desarrollado en los humedales de Ventanilla contribuirá a potencializar el crecimiento turístico sostenible con el tiempo y el surgimiento económico del distrito, lo cual fomentará la conciencia ambiental en la población y visitantes del lugar. Mediante las actividades ecológicas como el desarrollo de circuitos ecoturísticos sobre la observación de flora y fauna se logrará la protección del lugar 
difundiendo la importancia del valor natural que este atractivo posee. En las actividades se harán participes a los pobladores de la comunidad para contribuir con su mejora de las condiciones de vida.

Asimismo, para la elaboración del plan de ecoturismo, se apoyará de talleres aplicados a la población y visitantes sobre conciencia ambiental para evitar la contaminación en la zona, realizar esporádicamente campañas de limpieza mediante convocatorias voluntarias a colegios universidades. La difusión del lugar mediante una plataforma virtual e incentivar la participación de la población en la actividad artesanal con el trabajo de la planta del junco y carrizo que habita el humedal.

\section{Bibliografía}

Artaraz, M. (2002). Teoria de las tres dimensiones. Ecosistemas, 11(2), 2.

Bermejo, R. (2014). Desarrollo sostenible segun Brundtland a la sostenibilidad como biomimesis. Bilbao: Marra, S.L.

Crhoy. (Julio de 2018). Costa Rica lidera en ecoturismo. Costa Rica lidera como uno de los principales destinos ecoturísticos del mundo .

Criales, A. (2014). Centro regional para el aviturismo en la Albufera de medio mundo. (Tesis de licenciatura). Lima: Universidad San Martin de Porres.

Cuervo, R. (1967). El turismo como medio de Comunicación Humana. México: Departamento de Turismo del Gobierno de México.

Efe verde. (Noviembre de 2018). El aviturismo, sector en auge en el centro de Colombia. Efe verde.

Gobierno Regional del Callao. (2009). Plan Maestro 2009-2014. Área de Conservación Regional Humedales de Ventanilla. Obtenido de SERNANP: http://www.sernanp.gob.pe/planesmaestros

Hernández, R., Fernández, C., y Baptista, M. (2014). Metodología de la investigación. México: Interamericana editores, S.A

Hurtado, J. (2012). Metodología de la investigación. Caracas: Quiron Ediciones.

Mantero, J. (2004). Las dimensiones del desarrollo a propósito de 1 actividad turística. Aportes y transferencias, 1(28), 12. Obtenido de http://nulan.mdp.edu.ar/281/1/Apo2004a8v1pp1138.pdf

Massam, B. (2002). Quality of Life: Public Planning and Private Living. Pergamon(58), 141-227.

Ministerio de Comercio Exterior y Turismo, (2011). Guía para la formulación de proyectos de inversión exitosos. https://www.mincetur.gob.pe/wpcontent/uploads/documentos/turismo/proyectos_inversion_publica/instrumentos_metodol ogicos/Guia_Turismo_capitulo1.pdf

Organización Mundial de Turismo, (1993). Desarrollo sostenible del turismo. http://sdt.unwto.org/es/content/definicion

Panosso, A., \& Lohamann, G. (2012). Teoría del turismo: conceptos, modelos y sistemas. México: Trillas S.A.

PROMPERU (2018). Perú es nombrado en China como mejor destino del mundo para observación de aves https://www.promperu.gob.pe/Repos/pdf_novedades/26112018115624_404.pdf

Quintero, J. (2008). Turismo y desarrollo local en México: El caso del municipio de Cabo Corrientes en Jalisco. Guadalajara: Universidad de Guadalajara, Universidad complutense de Madrid. 
Secretaría de Turismo de La Nación, (2007). Guía de recomendaciones ambientales. http://www.ecopuerto.com/Bicentenario/informes/GUIARECOMENDAMBTURISMO.p df

The International Ecotourism Society, (2015). Ecotourism. https://ecotourism.org/what-isecotourism/

Ugaz, S. (2018). Ecoturismo como oportunidad de desarrollo sostenible del distrito de Jazán, departamento de Amazonas. (Tesis de licenciatura). Amazonas: Universidad Señor de Sipán.

Universidad Nacional Mayor de San Marcos. (2018). Medialab. Humedales de Ventanilla: Una lucha por sobrevir, pág. s/p.

Vásquez, A. (2000). Desarrollo económico local y descentralización: aproximación a un marco conceptual.

Obtenido

de https://repositorio.cepal.org/bitstream/handle/11362/31392/S00020088_es.pdf

Yarleque. (2004). La conservacion ambiental en estudiantes de educacion secundaria. Lima: Universidad Mayor de San Marcos. 